\title{
Study protocol for a controlled trial of an eHealth system utilising patient reported outcome measures for personalised treatment and care: PROMPT-Care 2.0
}

Afaf Girgis ${ }^{1,2^{*}}$ (D), Ivana Durcinoska ${ }^{1,2}$, Martha Gerges ${ }^{1,2}$, Nasreen Kaadan ${ }^{1,2,3}$, Anthony Arnold ${ }^{1,4}$, Joseph Descallar ${ }^{1,2,5}$, Geoff P. Delaney ${ }^{1,2,3}$ and on behalf of the PROMPT-Care Program Group

\begin{abstract}
Background: Routine assessment and clinical utilisation of patient-reported outcome (PRO) measures can lead to improved patient outcomes. The PROMPT-Care eHealth system facilitates PRO data capture from cancer patients, data linkage and retrieval to support clinical decisions, patient self-management, and shared care. Pilot testing demonstrated acceptability and feasibility of PROMPT-Care Version 1.0. This study aims to implement PROMPT-Care Version 2.0 and determine its efficacy in reducing emergency department (ED) presentations, and improving chemotherapy delivery and health service referrals, compared to usual care.

Methods: Groups eligible to participate in the intervention arm of this controlled trial are patients receiving cancer care (including follow-up). PROMPT-Care patients will complete monthly assessments (distress, symptoms, unmet needs) until voluntary withdrawal or death. In Version 1.0, the care team accessed patients' clinical feedback reports in 'real time' to guide their care, and patients received links to support their self-management, tailored to their PRO responses. Version 2.0 was extended to include: i) an additional alert system notifying the care team of ongoing unresolved clinical issues, ii) patient self-management resources, and iii) an auto-populated Treatment Summary and Survivorship Care Plan (SCP). The control population will be patients extracted from hospital databases of the general cancer patient population who were seen at the participating cancer therapy centres during the study period, with a ratio of 1:4 of intervention to control patients.

A minimum sample size of 1760 (352 intervention and 1408 control) patients will detect a 14\% reduction in the number of ED presentations (primary outcome) in the PROMPT-Care group compared with the control group. Intervention patients will provide feedback on system usability and value of the self-management materials; oncology staff will provide feedback on usefulness of PROMPT-Care reports, response to clinical alerts, impact on routine care, and usefulness of the SCPs; and GPs will provide feedback on the usefulness of the SCPs and attitudes towards shared-care models of survivorship care planning.
\end{abstract}

Discussion: This study will inform the PROMPT-Care system's impact on healthcare utilisation and utility as an alternative model for ongoing supportive care.

(Continued on next page)

\footnotetext{
*Correspondence: afaf.girgis@unsw.edu.au

${ }^{1}$ Centre for Oncology Education and Research Translation (CONCERT)

Ingham Institute for Applied Medical Research, Sydney, NSW, Australia

${ }^{2}$ South Western Sydney Clinical School, The University of New South Wales,

Sydney, Australia

Full list of author information is available at the end of the article
}

(c) The Author(s). 2018 Open Access This article is distributed under the terms of the Creative Commons Attribution 4.0 International License (http://creativecommons.org/licenses/by/4.0/), which permits unrestricted use, distribution, and reproduction in any medium, provided you give appropriate credit to the original author(s) and the source, provide a link to the Creative Commons license, and indicate if changes were made. The Creative Commons Public Domain Dedication waiver (http://creativecommons.org/publicdomain/zero/1.0/) applies to the data made available in this article, unless otherwise stated. 
(Continued from previous page)

Trial registration: Australian New Zealand Clinical Trials Registry (ACTRN12616000615482) on 12 th May 2016 (wwW. anzctr.org.au).

Keywords: Patient-reported outcomes (PROs), eHealth, Patient-centred care, Electronic health record, Selfmanagement, Risk-stratified care, Screening, Non-randomized control trial, Supportive care, Survivorship care

\section{Background}

Patient-reported outcomes (PROs) clearly place the patient's voice at the forefront of health care delivery [1], with systems to routinely collect and utilise PROs in clinical settings demonstrated to be feasible and acceptable [2-5]. Routinely screening for symptoms and other PROs and utilising these data to inform patient care has also been demonstrated to lead to significant improvements in patient outcomes and care indicators. In particular, reductions in emergency department visits $[6,7]$, longer tolerability of chemotherapy [6], improvements in both shortand long-term survival $[6,8]$, improved health related quality of life [9] and improved communication between patients and clinicians [9-11] have been documented in oncology settings. PROs have also been effectively used in non-oncology settings, including to inform surgical decisions in the orthopaedic setting $[12,13]$.

We have previously reported the development and acceptability and feasibility testing of an integrated PRO eHealth system, PROMPT-Care (Patient Reported Outcome Measures for Personalised Treatment and Care) [14, 15]. This system supports routine collection and analysis of cancer patients' PROs, real-time feedback of PRO results to their cancer care team to inform patient-centred care, and delivers evidence based self-management information to address patient reported problems. Our feasibility study demonstrated that the PROMPT-Care eHealth system is acceptable to the users, i.e. to the patients and cancer care team, and potentially feasible to implement in cancer centres [15]. Integration of the PRO measures into the hospital's point-of-care oncology information system (OIS), a key feature distinguishing PROMPT-Care from previous oncology-based eHealth systems, was hypothesised to enhance their relevance and usefulness in informing routine cancer care [16]. Our previous testing was not designed to inform the utility elements of the PROMPT-Care system or its efficacy. Therefore, this will be the primary purpose of the proposed study.

We have used the term patient in reference to all people diagnosed with cancer who are currently on treatment and in follow-up.

\section{Objective}

The overall objective of this study is to implement the PROMPT-Care 2.0 eHealth platform and determine its efficacy among cancer patients at four tertiary hospitals.
Specifically, this study will test whether web-based routine collection of PROs, combined with automated alerts to clinical teams and provision of patient self-management resources, result in reduced emergency department presentations, and improved chemotherapy delivery and health service referrals. The study will also evaluate system utility and potential benefits and barriers to PROMPT-Care implementation in routine care from both the patient and healthcare professional perspective.

\section{Methods/design \\ Setting}

The study is being undertaken in the cancer therapy centres of four participating hospitals, with oversight of the implementation undertaken by a clinical study lead: Liverpool Cancer Therapy Centre and Macarthur Cancer Therapy Centre (GD), Illawarra Cancer Care Centre and Shoalhaven Cancer Care Centre (AA).

\section{Ethics approval}

Ethics approval was obtained from the Human Research Ethics Committees of South Western Sydney, and Illawarra Shoalhaven Local Health Districts (Reference No. HREC/15/LPOOL/287).

\section{PROMPT-care intervention}

As previously reported $[14,15]$, the PROMPT-Care platform facilitates patients completing PRO measures online through standardised assessment tools using an electronic device (e.g. tablet, iPad, smart phone, or computer) and automatically converts these data into a format (HL7 messages) [17] that is transferred directly into the patient's point-of-care OIS in 'real time', following an automated matching verification process to ensure the correct record is populated. The point of care system used in this trial was Mosaiq ${ }^{\mathrm{Tm}}$ (Elekta Medical Systems, Sunnyvale, CA). PROs assessed include: Distress Thermometer (DT) and associated checklist [18], the Edmonton Symptom Assessment Scale (ESAS) [19], and the Supportive Care Needs Survey-Screening Tool 9 (SCNS-ST9) [20].

Feedback received during feasibility testing of pilot configuration for the PROMPT-Care system (Version 1.0) [15], highlighted additional patient and clinical team needs. As a result, Version 2.0 of the PROMPT-Care system has been extended to include the following 
elements: i) an additional alert system notifying the cancer care team of patients with ongoing unresolved issues, ii) tiered patient self-management resources, and iii) an auto-populated Treatment Summary and Survivorship Care Plan (SCP).

Version 2.0 of the PROMPT-Care intervention consists of three components (Fig. 1.):

1. Timely clinical care

Clinical feedback reports

Using previously reported algorithms [21], the uploaded PRO data is presented in a clinical feedback report (Fig. 2), which includes a basic one-page summary of the results of the most recent assessment, recommended clinical actions and suggested referrals, as well as a longitudinal report (Fig. 3) of patients' scores over time on the PRO scales. The reports are available 'real time' for clinical staff to review in the clinic with patients.

Clinical alerts

If the patient's scores on any of the PROs breach a predefined threshold on two consecutive assessments, an automated alert will be generated in the OIS, with an email received by a designated member of the cancer centre team, who will review the PROMPT-Care report and follow the care pathway agreed for that cancer centre.

\section{Tiered patient self-management}

Upon completing their PROMPT-Care assessment, patients will receive an email which directs them to a website containing tailored self-management information resources (Fig. 4) to address issues of concern they identified in their assessment. In response to patient feedback in the feasibility study [15], the PROMPT-Care system was modified to provide patients with a tiered approach to their self-management support. The first time a patient breaches a PRO item, they will receive a link to generic information resources via one of the four distinct domain-specific webpages hosted on the Cancer Institute NSW eviQ website: emotional well-being, physical well-being, social/family well-being, practical problems. Patients who do not breach any items will receive a link to the "maintaining well-being" page, to support

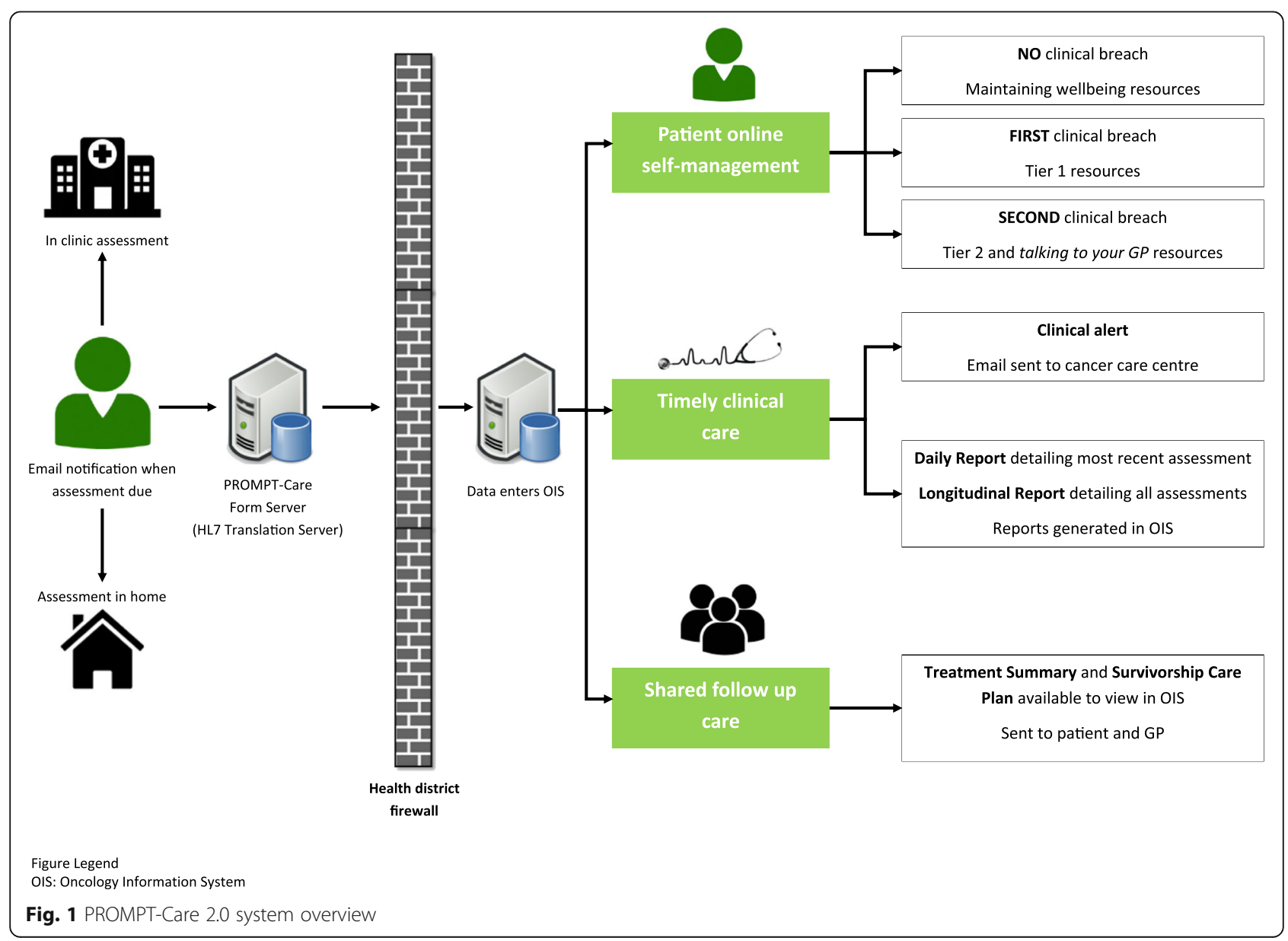




\section{Liverpool Cancer Therapy Centre}

\section{PROMPTMCare}

\begin{tabular}{lll}
\hline Patient Name: ZZZTEST, NK3 & MRN: 01234MRN & DOB: 01/01/1900 \\
\hline $\begin{array}{l}\text { THIS PATIENT HAS A HIGH DISTRESS SCORE. Review remainder of report for potential causes of this distress and action the specific } \\
\text { recommendations as detailed }\end{array}$ & Recommendation \\
\hline $\begin{array}{l}\text { Emotional } \\
\text { Fears of cancer spreading }\end{array}$ & $\begin{array}{l}\text { Consider reasons for concern and compare to previous assessments (if available). If results are } \\
\text { identical or increased, refer to social work OR psychology services for further assessment and } \\
\text { care. }\end{array}$ \\
\hline Physical & $\begin{array}{l}\text { Recommendation } \\
\text { Chortness of breath }\end{array}$ & $\begin{array}{l}\text { Clinically address as appropriate OR refer to relevant medical specialist OR allied health } \\
\text { professional for further assessment and care } \\
\text { Consider reasons for concern and, if required, refer to social work OR psychology services OR to } \\
\text { centre-specific or community services for further assessment and care }\end{array}$ \\
Appearance & $\begin{array}{l}\text { Recommendation } \\
\text { Discuss treatment concerns with patient. Consider reasons for concern and, if required, refer to } \\
\text { social work OR psychology services for further assessment and care }\end{array}$ \\
\hline Practical & $\begin{array}{l}\text { Recommendation } \\
\text { Consider reasons for concern and, if required, refer to relevant medical specialist OR social work } \\
\text { OR psychology services for further assessment and care }\end{array}$ \\
\hline Social/Family & \\
Ability to have children &
\end{tabular}

1. Distress (Distress Thermometer \& Problem Checklist)

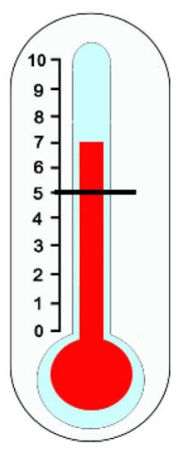

\begin{tabular}{|l|l|}
\hline Practical problems: & Emotional problems: \\
\hline Ghildcare & Depression \\
\hline Housing & Fears \\
\hline Insurance/financial & Nervousness \\
\hline Transportation & Sadness \\
\hline Work/School & Worry \\
\hline Treatment decisions & Loss of interest in usual activities \\
\hline Other: & Goping with illness \\
\hline Spiritual/Religious & Losing Hope \\
\hline Family problems: & \multicolumn{2}{|l}{} \\
\hline Dealing with partner & Dealing with Children \\
\hline Ability to have children & Lack of support from family \& \\
\hline Family health issues & friends \\
\hline
\end{tabular}

\begin{tabular}{|l|l|}
\hline Physical problems: \\
\hline Appearance & Indigestion \\
\hline Bathing/dressing & Memory/concentration \\
\hline Breathing & Aouth sores \\
\hline Changes in urination & Nausea \\
\hline Constipation & Nose dry/congested \\
\hline Biarrhoea & Pain \\
\hline Eating & Sexual \\
\hline Fatigue & Skin drylitchy \\
\hline Feeling swollen & Sleep \\
\hline Fevers & Substance abuse \\
\hline Getting around & Fingling in hands/feet \\
\hline &
\end{tabular}

2. Symptoms (ESAS)

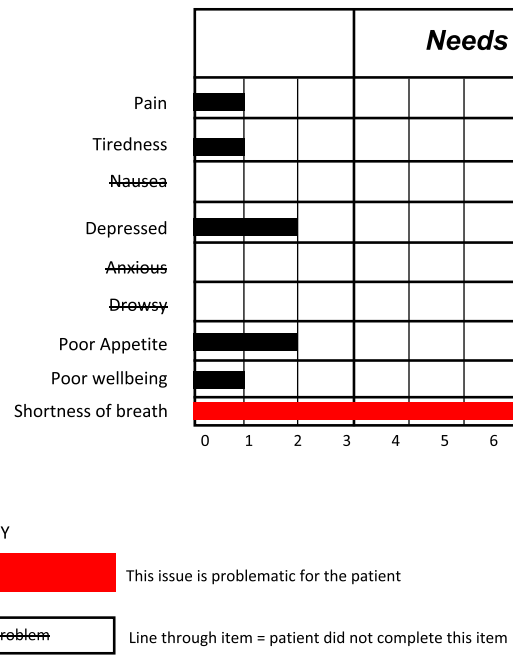

3. Unmet Needs (SCNS-ST9)

\begin{tabular}{|c|c|}
\hline Need & Issue \\
\hline \multirow[t]{2}{*}{ Psychological } & $\begin{array}{l}\text { Fears about the cancer } \\
\text { spreading }\end{array}$ \\
\hline & Uncertainty about the future \\
\hline \multirow[t]{2}{*}{$\begin{array}{l}\text { Health system and } \\
\text { information }\end{array}$} & $\begin{array}{l}\text { Being informed about your test } \\
\text { results as soon as feasible }\end{array}$ \\
\hline & $\begin{array}{l}\text { Being informed about things you } \\
\text { can do to help yourself to get } \\
\text { well }\end{array}$ \\
\hline \multirow{2}{*}{$\begin{array}{l}\text { Physical and daily } \\
\text { living }\end{array}$} & Lack of energy/tiredness \\
\hline & $\begin{array}{l}\text { Not being able to do the things } \\
\text { you used to do }\end{array}$ \\
\hline \multirow[t]{2}{*}{$\begin{array}{l}\text { Patient care and } \\
\text { support }\end{array}$} & $\begin{array}{l}\text { Reassurance by medical staff } \\
\text { that the way you feel is normal }\end{array}$ \\
\hline & $\begin{array}{l}\text { Hospital staff acknowledging } \\
\text { and show sensitivity to your } \\
\text { feelings and emotional needs }\end{array}$ \\
\hline Sexuality & $\begin{array}{l}\text { Changes in sexual } \\
\text { relationships }\end{array}$ \\
\hline
\end{tabular}

Fig. 2 Sample clinical feedback report 
Liverpool Cancer Therapy Centre

PROMPT»Care

Patient Name: ZZZTEST, NK3

MRN: 01234MRN

DOB: 01/01/1900

1. Distress (Distress Thermometer \& Problem Checklist)

Date

$10 / 03 / 2016$

$10 / 03 / 2016$

$20 / 04 / 2016$

$20 / 07 / 2016$

$11 / 08 / 2016$

$04 / 10 / 2016$

$28 / 10 / 2016$

$02 / 08 / 2017$

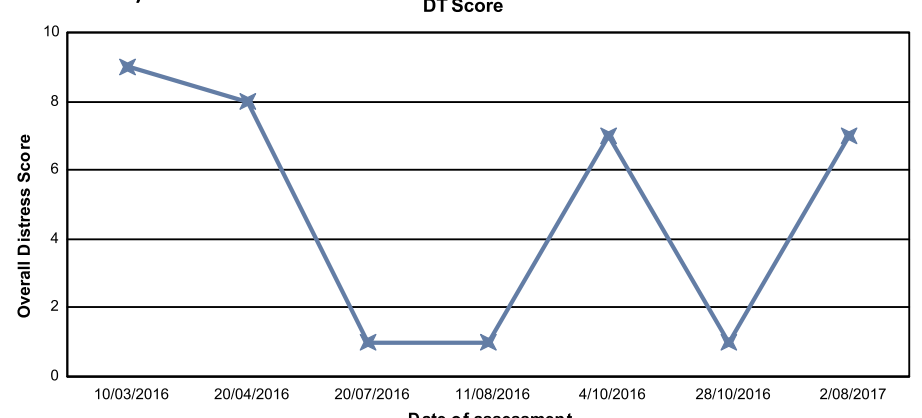

\begin{tabular}{|c|c|c|c|c|c|c|c|c|c|c|c|c|c|c|c|c|c|c|c|c|}
\hline \multirow[b]{2}{*}{ Date } & \multicolumn{6}{|c|}{ Practical Problems } & \multicolumn{5}{|c|}{ Family Problems } & \multicolumn{8}{|c|}{ Emotional Problems } & \multirow[b]{2}{*}{ 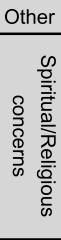 } \\
\hline & 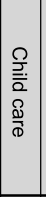 & 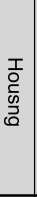 & 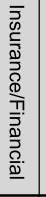 & 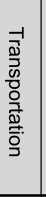 & 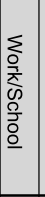 & 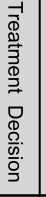 & 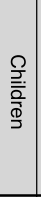 & 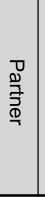 & 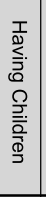 & 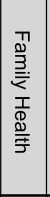 & 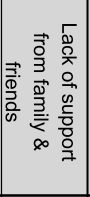 & $\begin{array}{l}\text { D } \\
\frac{D}{0} \\
\frac{0}{0} \\
0 \\
\frac{\infty}{0}\end{array}$ & $\begin{array}{l}7 \\
\mathbb{8} \\
\stackrel{M}{\omega}\end{array}$ & 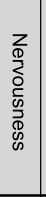 & 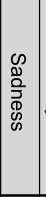 & $\sum_{0}$ & 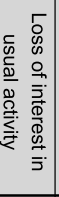 & 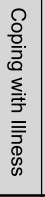 & 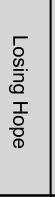 & \\
\hline $11 / 03 / 2016$ & ---- & --- & --- & --- & \begin{tabular}{|c|}
--- \\
\end{tabular} & --- & --- & ---[ & & \begin{tabular}{|l|l}
--- \\
\end{tabular} & ---- & ---- & & --- & & \begin{tabular}{|c|}
---- \\
\end{tabular} & \begin{tabular}{|l|}
--- \\
\end{tabular} & ---- & ---- & --- \\
\hline $16 / 03 / 2016$ & \begin{tabular}{|l|}
--- \\
\end{tabular} & - & $-\cdots$ & --- & \begin{tabular}{|l|}
--- \\
\end{tabular} & --- & $-\cdots$ & ---1 & $-\cdots$ & \begin{tabular}{|l|}
--- \\
\end{tabular} & --- & --- & & $---{ }_{-1}$ & $\mid--\cdot$ & $\mid--$ & \begin{tabular}{|l|}
--- \\
\end{tabular} & --- & --- & --- \\
\hline 22/03/2016 & 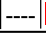 & -- & $---\mid$ & $---\mid$ & 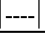 & $|---|$ & $---\mid$ & $---\mid$ & $---\mid$ & $|---|$ & ---- & --- & -1 & $---\mid$ & $|---|$ & |---| & $---\mid$ & $---\mid$ & & ---- \\
\hline
\end{tabular}

\begin{tabular}{|c|c|c|c|c|c|c|c|c|c|c|c|c|c|c|c|c|c|c|c|c|c|c|}
\hline \multicolumn{23}{|c|}{ Physical Problems } \\
\hline Date & 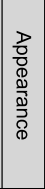 & 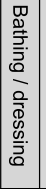 & 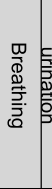 & 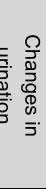 & 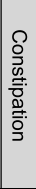 & 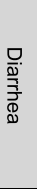 & $\begin{array}{l}m \\
\mathbb{N} \\
\vec{\Xi} \\
\mathbb{0}\end{array}$ & 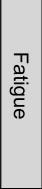 & 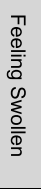 & $\begin{array}{l}\frac{T}{\Phi} \\
\frac{\Phi}{\omega}\end{array}$ & 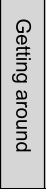 & $\begin{array}{l}\bar{z} \\
\overline{2} \\
0 \\
0 \\
0 \\
\overline{0} \\
\overline{0}\end{array}$ & 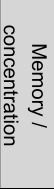 & $\begin{array}{l}3 \\
0 \\
\frac{1}{5} \\
\frac{1}{5} \\
0 \\
\frac{0}{8} \\
0\end{array}$ & $\begin{array}{l}z \\
\mathbb{2} \\
\mathcal{D} \\
\infty \\
\mathbb{D} \\
\mathbb{D}\end{array}$ & 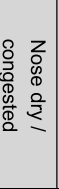 & 总 & $\begin{array}{l}\mathscr{W} \\
\mathbb{D} \\
\underset{x}{\mathbb{2}} \\
\underline{\mathbb{N}}\end{array}$ & 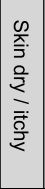 & \begin{tabular}{|l}
$\frac{\omega}{\mathbb{D}}$ \\
$\frac{\mathbb{D}}{\delta}$
\end{tabular} & 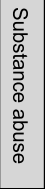 & 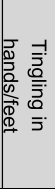 \\
\hline $11 / 03 / 2016$ & --- & \begin{tabular}{|l|}
--- \\
\end{tabular} & --- & --- & --- & --- & \begin{tabular}{|l|}
--- \\
\end{tabular} & --- & --- & - & \begin{tabular}{|l|}
--- \\
--1
\end{tabular} & \begin{tabular}{|l|}
--- \\
\end{tabular} & -.. & --- & --- & --- & \begin{tabular}{|l|}
--- \\
--1
\end{tabular} & $-{ }_{-1}$ & \begin{tabular}{|l|}
--- \\
\end{tabular} & \begin{tabular}{|l|}
--- \\
--
\end{tabular} & & ---- \\
\hline $16 / 03 / 2016$ & --- & --- & --- & --- & -- & --- & --- & --- & --- & --- & --- & --- & --- & --- & --- & --- & --- & ---- & --- & --- & & --- \\
\hline $20 / 04 / 2016$ & & ---- | & |--- & --- & |--- & ---- & $|---|$ & $---\left.\right|^{\prime}$ & |--- & --- & ---- | & $|---|$ & |--- & --- & ---- & |--- & $|---|$ & --- & $|---|$ & |--- | & $|---|$ & |--- \\
\hline
\end{tabular}

\section{Symptoms (Edmonton Symptom Assessment Scale)}

\begin{tabular}{|l|c|c|c|c|c|c|c|c|c|}
\hline Date & $\begin{array}{c}\text { Pain } \\
/ 10\end{array}$ & $\begin{array}{c}\text { Tiredness } \\
/ 10\end{array}$ & $\begin{array}{c}\text { Nausea } \\
/ 10\end{array}$ & $\begin{array}{c}\text { Depressed } \\
/ 10\end{array}$ & $\begin{array}{c}\text { Anxious } \\
/ 10\end{array}$ & $\begin{array}{c}\text { Drowsy } \\
/ 10\end{array}$ & $\begin{array}{c}\text { Appetite } \\
/ 10\end{array}$ & $\begin{array}{c}\text { Wellbeing } \\
/ 10\end{array}$ & $\begin{array}{c}\text { Shortness of } \\
\text { Breath } / 10\end{array}$ \\
\hline $\mathbf{3 1 / 0 3 / 2 0 1 6}$ & ---- & ---- & ---- & ---- & ---- & ---- & 0 & ---- & ---- \\
\hline $\mathbf{2 0 / 0 4 / 2 0 1 6}$ & 2 & ---- & 0 & 0 & 2 & ---- & ---- & 3 & 7 \\
\hline $\mathbf{2 0 / 0 7 / 2 0 1 6}$ & ---- & ---- & 0 & 7 & ---- & ---- & ---- & ---- & ---- \\
\hline
\end{tabular}

\section{Unmet Needs (Supportive Care Needs Survey ST9)}

\begin{tabular}{|c|c|c|c|c|c|c|c|c|c|}
\hline \multicolumn{10}{|c|}{ No Need (1=N/A; 2= Satifisfied) | Some Need (3=Low Need; 4=Moderate Need; 5=High Need) } \\
\hline & \multicolumn{2}{|c|}{ Psychological Need } & \multicolumn{2}{|c|}{$\begin{array}{l}\text { Health System \& } \\
\text { Information Need }\end{array}$} & \multicolumn{2}{|c|}{ Daily Living Need } & \multicolumn{2}{|c|}{ Patient Care Need } & \multirow{2}{*}{$\begin{array}{c}\text { Sexuality } \\
\text { Need }\end{array}$} \\
\hline Date & $\begin{array}{l}\text { Fears about } \\
\text { the cancer } \\
\text { spreading }\end{array}$ & $\begin{array}{c}\text { Uncertainty } \\
\text { about the } \\
\text { future }\end{array}$ & $\begin{array}{c}\text { Being } \\
\text { informed } \\
\text { about your } \\
\text { test results } \\
\text { as soon as } \\
\text { feasible }\end{array}$ & $\begin{array}{l}\text { Being } \\
\text { informed } \\
\text { about } \\
\text { things you } \\
\text { can do to } \\
\text { help }\end{array}$ & $\begin{array}{l}\text { Lack of } \\
\text { energy / } \\
\text { tiredness }\end{array}$ & $\begin{array}{l}\text { Not being } \\
\text { able to do } \\
\text { the things } \\
\text { you used to }\end{array}$ & $\begin{array}{c}\text { Reassuranc } \\
\text { e by medical } \\
\text { staff that the } \\
\text { way you feel } \\
\text { is normal }\end{array}$ & $\begin{array}{l}\text { Hospital staff } \\
\text { acknowledgin } \\
\text { g, and } \\
\text { showing } \\
\text { sensitivity to } \\
\text { your feelings }\end{array}$ & \\
\hline $20 / 04 / 2016$ & 4 & 1 & ---- & 2 & 1 & 1 & 2 & 1 & 2 \\
\hline $20 / 07 / 2016$ & 4 & --- & --- & --- & --- & --- & --- & --- & --- \\
\hline
\end{tabular}

Fig. 3 Sample longitudinal feedback report 


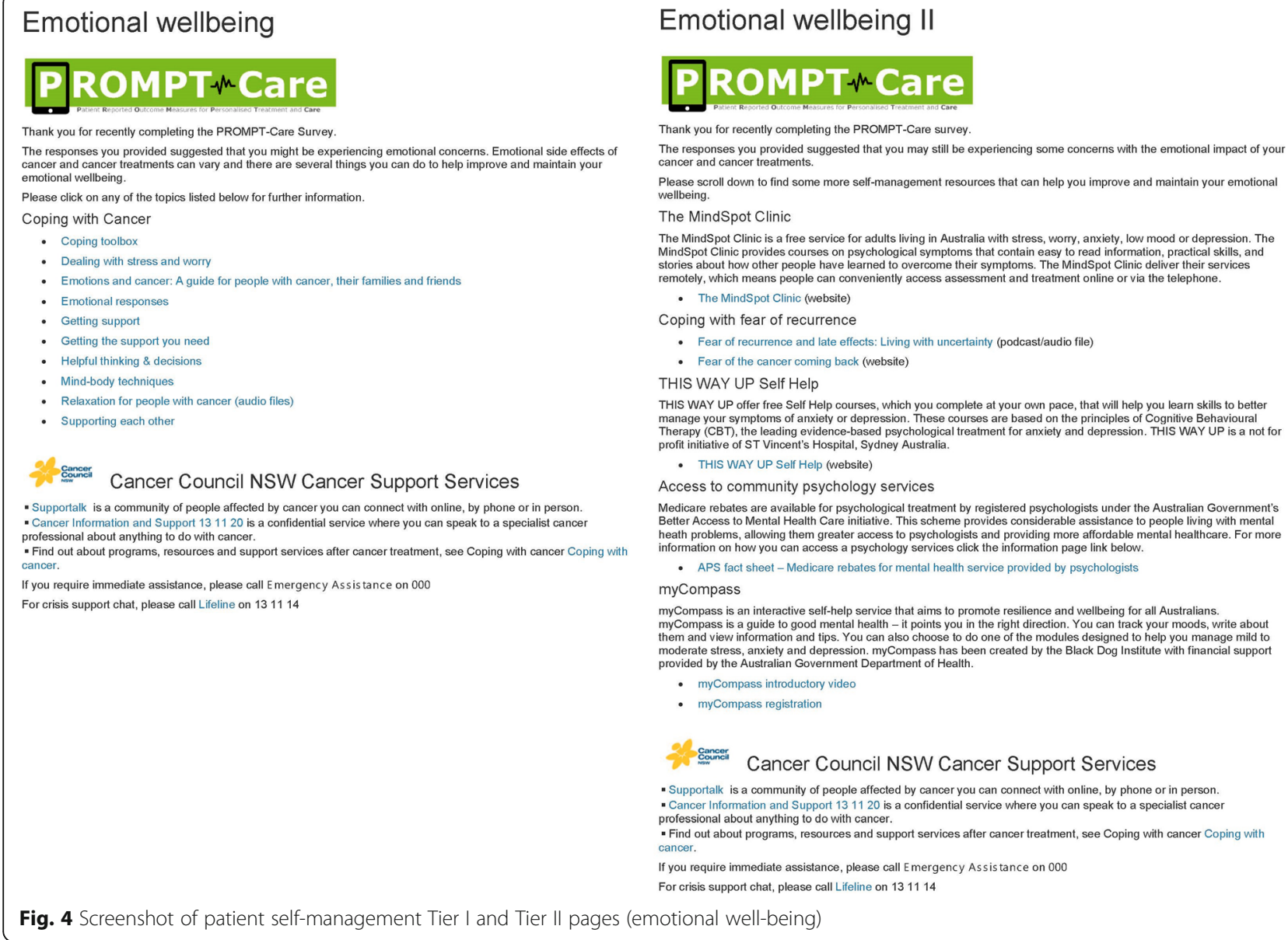

Fig. 4 Screenshot of patient self-management Tier I and Tier II pages (emotional well-being)

Eligible patients are people who are either currently receiving cancer care (including follow-up care) or have recently been diagnosed with cancer and are scheduled to commence cancer treatment at one of the four participating sites. Eligibility criteria include a confirmed diagnosis of cancer, age 18 years or over, cognitively able to provide informed consent and understand the surveys, and ability to complete the survey in English. Exclusion criteria are having a diagnosis of a blood cancer and not having access to the Internet outside of the clinic.

\section{Oncology staff}

All staff who provide care in the oncology departments at the participating hospitals are eligible to participate.

\section{General practitioners (GPs)}

All GPs nominated by a participating patient as their primary care provider will be eligible to participate. 


\section{Control population}

A list of potential control individuals (minimum $n=$ 1408) will be extracted from hospital databases of the general cancer patient population who were seen at the participating cancer therapy centres during the study period.

\section{Procedure}

\section{Oncology staff engagement and consent}

Oncology staff (including specialists and nurses) will be invited to participate via email and sent an introductory summary, information sheet and consent form. Treating clinicians are asked to provide permission for research staff to contact their patients, and consent to participate in an evaluation interview at study close. Consenting oncology staff will receive training resources and participate in orientation sessions on how to use the PROMPT-Care system in routine clinical practice at study start along with refresher resources and orientation throughout the trial as needed.

\section{Patient identification and consent}

Participating clinicians will review their clinic lists to identify eligible patients who have appointments scheduled within the coming two (2) months. Two patient recruitment approaches will be utilised to achieve a similar proportion of both patients in active treatment and those in follow-up care.

In clinic Eligible patients attending a cancer care clinic will be invited to participate by a member of the clinical trial team in the waiting area. The trial research member will explain the study in detail and provide patients with a study pack containing a letter of invitation, participant information and consent form, demographics survey and reply-paid envelope. Consenting patients will be asked to complete a consent form and their first PROMPT-Care assessment just prior to their appointment.
Mail-out For patients who require additional time to consider participation, or those in follow-up who do not have regular clinic appointments, research staff will mail out a study pack inviting them to participate, then follow up by phone to answer any questions and provide additional information, as required.

\section{Assessment completion}

Patients will complete PROMPT-Care assessments on a monthly basis and will be followed up for a minimum of four months. Patients will have the option to complete assessments either at the hospital in clinic, their home or any public community location. Patients completing assessments in clinic will be provided with a tablet device in the waiting area just prior to their scheduled appointment. Patients who complete assessments from home or in community locations will receive an email containing the survey link three days before their assessment is due.

\section{Measures \\ Outcomes}

The primary outcome is number of emergency department (ED) presentations observed during the study period. Details about ED presentation dates, reason for presentation and any resulting admissions will be extracted from the electronic medical record (EMR) (Table 1).

The secondary outcomes are time receiving active chemotherapy and referrals to allied health services. Details of planned and actual chemotherapy regimens as well as any toxicities and changes to treatment delivery will be extracted from the OIS. Date, reason and number of referrals to allied health services will be tabulated and extracted from the medical record and OIS.

\section{Patient clinical and socio-demographic characteristics}

Upon consenting, participants will complete a questionnaire about socio-demographics including: marital status, education level, employment status and language spoken at home. Additional demographics (eg. age,

Table 1 Primary and secondary outcome data collected

\begin{tabular}{|c|c|c|}
\hline Data & Description of data & Source of data \\
\hline \multicolumn{3}{|l|}{ Primary Outcomes } \\
\hline Emergency Department (ED) presentations & $\begin{array}{l}\text { - ED presentations (date, number of visits) } \\
\text { - Length of stay } \\
\text { - Reason for presentation }\end{array}$ & $\begin{array}{l}\text { Extracted from electronic medical } \\
\text { record (EMR) }\end{array}$ \\
\hline \multicolumn{3}{|l|}{ Secondary Outcomes } \\
\hline Time on chemotherapy & $\begin{array}{l}\text { - Planned chemotherapy regimen } \\
\text { - Actual regimen start and end date } \\
\text { - Toxicities, changes to treatment delivery and reasons }\end{array}$ & $\begin{array}{l}\text { Extracted from oncology information } \\
\text { system (OIS) }\end{array}$ \\
\hline Referral to allied health services & $\begin{array}{l}\text { - Date of referral and allied health service type } \\
\text { - Reason for referral eg. emotional distress, case } \\
\text { management etc. }\end{array}$ & Extracted from EMR \& OIS \\
\hline
\end{tabular}


gender and need for interpreter) and clinical characteristics such as date of diagnosis, site (ICD-10), stage (TNM classification) and treatment details will be extracted from the OIS.

\section{System utility evaluation}

Patient evaluation Patients will complete periodic online progress evaluation surveys following the completion of their third, sixth and ninth PROMPT-Care assessments. Patients will be asked about the usability of the system, preferences for timing of completing PROMPT-Care assessments, satisfaction and usefulness of the system, suitability and value of the self-management materials, and suggestions for further refinement. A sub-set of patients (approximately 10-20 patients) will also be invited to participate in semi-structured interviews at study completion in order to further explore themes identified in the evaluation surveys.

Healthcare professional evaluation Participating oncology staff and GPs will be invited to participate in evaluation surveys and semi-structured interviews at study completion. Oncology staff will be asked questions about, how they used the PROMPT-Care reports in clinical practice and their usefulness, their response to the clinical alerts, how PROMPT-Care impacted routine care, and their views on the Treatment Summary and SCPs. GP data will be analysed to evaluate the content and suitability of the Treatment Summary and SCPs. It will also be used to gauge attitudes towards shared-care models of survivorship care planning.

System usage statistics Data on the use of the PROMPT-Care system will be extracted from the OIS and evaluated to inform: frequency of report usage, clinical alert activity, assessment data transfer, and IT system functioning. User and technical system errors will also be monitored by research staff and recorded in an error $\log$ of IT issues and associated resolutions e.g. firewall upgrades, server downtime, participant report of IT problems completing assessments or accessing resource webpages. Patient interaction with and use of the self-management resources will be analysed by Google Analytics [22] and ClickMeter [23] over time. Google analytics will be used to gather data on the number of users and views of the domain-specific resource webpages (eg. emotional, physical, social/family, maintaining well-being, and practical problems), whereas ClickMeter will be used to track clicks into the individual resources $(n=114)$, sitting within each domain page. System usage data will be summarised using simple descriptive statistics and will be presented as counts, mean scores, standard deviations and percentages.

\section{Sample size}

A minimum sample size of 1760 (352 intervention and 1408 control) patients is required to detect a $14 \%$ reduction in the number of ED presentations in the PROMPT-Care group compared with the control group. This is based on the assumed rate of ED presentations being 1.4 visits per patient during the study period, a 1:4 ratio of PROMPT-Care to control group patients, $80 \%$ power and $5 \%$ statistical significance.

\section{Statistical analyses}

Descriptive statistics will be generated for all socio-demographic and clinical characteristics, and outcome measures. A multivariable Poisson or negative binomial regression (depending on over-dispersion) will be used to determine whether the rates of ED presentations were different between the PROMPT-Care and control groups adjusting for covariates (such as age, sex, stage of disease, and treatment status). Number of referrals to allied health services will be analysed similarly. Multivariable Cox proportional hazards model will be used to analyse length of time from start to end of chemotherapy adjusting for covariates.

\section{Qualitative analysis}

Interviews with patients and health professionals will be audio-recorded, transcribed verbatim, and analysed using thematic analysis [24]. Two researchers will independently read the transcripts and generate initial codes. Identified codes will then be collated into emerging themes. Themes will then be refined, with discrepancies resolved through discussion and consensus.

\section{Discussion}

To date, the impact of collecting and utilising PROs in the oncology setting have been studies in defined groups of patients. The results from this study will contribute important new evidence to the literature, with its inclusion of a broad population of patients who are currently undergoing cancer treatment or are in follow-up, and patients with a wide range of tumour types. PROMPT-Care Version 1.0 has previously been demonstrated to be feasible and acceptable $[14,15]$. This project will provide evidence regarding the impact of the expanded and improved PROMPT-Care Version 2.0 system on healthcare utilisation, including emergency department presentations, chemotherapy adherence and referral to allied health services; the acceptability of the tailored, stepped self -management resources; and usefulness of newly introduced strategies to facilitate shared follow-up care - the Treatment Summary and SCPs.

This information will be used to guide further revisions of the PROMPT-Care system and aid its wider implementation in other cancer centres in Australia; and inform its potential as an alternative model of providing 
ongoing patient supportive care. The resulting eHealth platform will be an evidence-informed tool which supports and enables cancer patients to achieve and maintain improved well-being and better cancer outcomes.

\author{
Abbreviations \\ CINSW: Cancer Institute NSW; DT: Distress Thermometer; ED: Emergency \\ Department; EMR: Electronic Medical Record; ESAS: Edmonton Symptom \\ Assessment Scale; GP: General Practitioner; MRN: Medical Record Number; \\ OIS: Oncology Information System; PRO: Patient Reported Outcome; \\ PROMPT-Care : Patient Reported Outcome Measures for Personalised \\ Treatment and Care; SCNS-ST9: Supportive Care Needs Survey - Screening \\ Tool 9 items; SCP: Survivorship Care Plan; TNM: Tumour, node and \\ metastases
}

\section{Acknowledgements}

The PROMPT-Care Program Group also includes the following authors: Sandra Avery, Martin Carolan, Stephen Della-Fiorentina, Kenneth Masters, Andrew Miller, Weng Ng, Tiffany Sandell, Thomas T Tran, Janelle V Levesque. The PROMPT-Care team includes clinicians and researchers from the South Western Sydney and Illawarra Shoalhaven Local Health Districts; and the Clinical and Technical Advisory Groups include more than 40 members from Local Health Districts across NSW as well as from the CINSW. Their significant input is gratefully acknowledged. We also wish to thank the patients who generously contributed their time and input to the development of PROMPT-Care.

\section{Funding}

Funding was provided by the Cancer Institute NSW, Bupa Health Foundation and the Wollondilly Health Alliance. CINSW staff participated in each of the project Advisory Groups, based on their expertise, thereby providing expert input into the development and content of the PROMPT-Care system. The funding bodies were not involved in the overall design of the study nor in the writing of this manuscript.

\section{Authors' contributions}

All authors were involved in design of the clinical trial. AG and GD obtained study funding. AG, GD, ID, NK, AA, JD, and the PROMPT-Care Program Group contributed to the design of the study. AG, ID, and MG are responsible for the implementation of the trial. All authors contributed to and approved the final manuscript.

\section{Ethics approval and consent to participate}

Ethics approval was obtained from the Human Research Ethics Committees of South Western Sydney, and Illawarra Shoalhaven Local Health Districts, with site-specific governance approvals obtained for Liverpool, Campbelltown, Wollongong and Shoalhaven Hospitals.

Participants will be provided with a study pack containing a letter of invitation, participant information sheet and consent form, demographics survey and reply-paid envelope. Written informed consent (return of completed consent form) will be obtained from all participants. No participant will start the trial until consent is received.

\section{Consent for publication}

Not applicable.

\section{Competing interests}

The authors declare that they have no competing interests.

\section{Publisher's Note}

Springer Nature remains neutral with regard to jurisdictional claims in published maps and institutional affiliations.

\section{Author details}

${ }^{1}$ Centre for Oncology Education and Research Translation (CONCERT) Ingham Institute for Applied Medical Research, Sydney, NSW, Australia. ${ }^{2}$ South Western Sydney Clinical School, The University of New South Wales, Sydney, Australia. ${ }^{3}$ Liverpool Cancer Therapy Centre, Liverpool Hospital, Liverpool, NSW, Australia. ${ }^{4}$ Illawarra Cancer Care Centre, Wollongong
Hospital, Wollongong, NSW, Australia. ${ }^{5}$ Department of Statistics, Macquarie University, Sydney, NSW, Australia.

Received: 11 January 2018 Accepted: 7 August 2018

Published online: 23 August 2018

\section{References}

1. Baumhauer JF. Patient-reported outcomes - are they living up to their potential? N Engl J Med. 2017;377(1):6-9.

2. Chen J, Ou L, Hollis SJ. A systematic review of the impact of routine collection of patient reported outcome measures on patients, providers and health organisations in an oncologic setting. BMC Health Serv Res. 2013;13:211.

3. Ristevski E, Regan M, Jones R, Breen S, Batson A, McGrail M. Cancer patient and clinician acceptability and feasibility of a supportive care screening and referral process. Health Expect. 2015;18(3):406-18.

4. Mitchell AJ. Screening for cancer-related distress: when is implementation successful and when is it unsuccessful? Acta Oncol. 2013;52(2):216-24.

5. Mitchell AJ, Vahabzadeh A, Magruder K. Screening for distress and depression in cancer settings: 10 lessons from 40 years of primary-care research. Psychooncology. 2011;20(6):572-84.

6. Basch E, Deal AM, Kris MG, Scher HI, Hudis CA, Sabbatini P, Rogak L, Bennett AV, Dueck AC, Atkinson TM, et al. Symptom Monitoring With PatientReported Outcomes During Routine Cancer Treatment: A Randomized Controlled Trial. J Clin Oncol. 2016;34(6):557-+.

7. Barbera L, Sutradhar R, Howell D, Sussman J, Seow H, Dudgeon D, Atzema C, Earle C, Husain A, Liu Y, et al. Does routine symptom screening with ESAS decrease ED visits in breast cancer patients undergoing adjuvant chemotherapy? Support Care Cancer. 2015;23(10):3025-32.

8. Basch E, Deal AM, Dueck AC, et al. Overall survival results of a trial assessing patient-reported outcomes for symptom monitoring during routine cancer treatment. JAMA. 2017:318(2):197-8. https://doi.org/10.1001/jama.2017.7156.

9. Velikova G, Booth L, Smith AB, Brown PM, Lynch P, Brown JM, Selby PJ. Measuring quality of life in routine oncology practice improves communication and patient well-being: a randomized controlled trial. J Clin Oncol. 2004;22(4):714-24.

10. Carlson LE, Waller A, Groff SL, Bultz BD. Screening for distress, the sixth vital sign, in lung cancer patients: effects on pain, fatigue, and common problems-secondary outcomes of a randomized controlled trial. Psychooncology. 2013;22(8):1880-8. https://doi.org/10.1002/pon.3223.

11. Yang L, Manhas D, Howard A, Olson R. Patient-reported outcome use in oncology: a systematic review of the impact on patient-clinician communication. Support Care Cancer. 2018;26(1):41-60.

12. Cella D, Yount $S$, Rothrock N, Gershon R, Cook K, Reeve B, Ader D, Fries JF, Bruce B, Rose M. The patient-reported outcomes measurement information system (PROMIS): progress of an $\mathrm{NIH}$ roadmap cooperative group during its first two years. Med Care. 2007:45(5 Suppl 1):S3-S11.

13. Papuga MO, Beck CA, Kates SL, Schwarz EM, Maloney MD. Validation of GAITRite and PROMIS as high-throughput physical function outcome measures following ACL reconstruction. J Orthop Res. 2014;32(6):793-801.

14. Girgis A, Delaney G, Arnold A, Miller AA, Levesque JV, Kaadan N, Carolan M, Cook N, Masters K, Tran TT, et al. Development and feasibility testing of PROMPT-care, an eHealth system for collection and use of patient-reported outcome measures for personalized treatment and care: a study protocol. JMIR Res Protoc. 2016;5(4):e227.

15. Girgis A, Durcinoska I, Levesque JV, Gerges M, Sandell T, Arnold A, Delaney GP. eHealth system for collecting and utilizing patient reported outcome measures for personalized treatment and care (PROMPT-care) among Cancer patients: mixed methods approach to evaluate feasibility and acceptability, J Med Internet Res, 2017:19(10):e330.

16. Jensen RE, Snyder CF, Abernethy AP, Basch E, Potosky AL, Roberts AC, Loeffler DR, Reeve BB. Review of Electronic Patient-Reported Outcomes Systems Used in Cancer Clinical Care. J Oncol Pract. 2014;10(4):e215-22. https://doi.org/10.1200/JOP.2013.001067.

17. Schuler T, Miller AA. PROsaiq: a smart device-based and EMR-integrated system for patient-reported outcome measurement in routine cancer care. J Rad Onco Inf. 2014:6(1):84-104.

18. National Comprehensive Cancer Network. NCCN Clinical Guidelines in Oncology: Distress Management, Version 2. 2018. https://www.nccn.org/ professionals/physician_gls/pdf/distress.pdf. Accessed 13 Aug 2018.

19. Richardson LA, Jones GW. A review of the reliability and validity of the Edmonton symptom assessment system. Curr Oncol. 2009;16(1):55. 
20. Girgis A, Stojanovski E, Boyes A, King M, Lecathelinais C. The next generation of the supportive care needs survey: a brief screening tool for administration in the clinical oncology setting. Psychooncology. 2012;21(8): 827-35.

21. Girgis A, Durcinoska I, Koh E-S, Ng W, Arnold, A, Avery S, Carolan M, Della-Fiorentina S, Kaadan N, Vardy J, Dhillon H, Miller A, Delaney GP. Development of health pathways to standardise cancer care pathways informed by patient-reported outcomes and clinical practice guidelines. JCO Clinical Cancer Informatics 2018. https://doi.org/10.1200/CCl.18.00024

22. Google Analytics Solutions: Marketing Analytics \& Measurement [https:// www.google.com/analytics/\#?modal_active=none].

23. ClickMeter: Link tracking, affiliate tracking, conversion tracking [https://www. clickmeter.com/].

24. Guest G, MacQueen KM, Namey EE. Applied thematic analysis. Thousand Oaks, CA: SAGE Publications Ltd; 2012

Ready to submit your research? Choose BMC and benefit from:

- fast, convenient online submission

- thorough peer review by experienced researchers in your field

- rapid publication on acceptance

- support for research data, including large and complex data types

- gold Open Access which fosters wider collaboration and increased citations

- maximum visibility for your research: over $100 \mathrm{M}$ website views per year

At $\mathrm{BMC}$, research is always in progress.

Learn more biomedcentral.com/submissions 